\title{
Next Wave of Bio-Therapy and its Applications
}

\section{Sanjeev K Gupta*}

Advanced Biotech Lab, Ipca Laboratories Ltd., Kandivli (W), Mumbai

*Corresponding Author: Sanjeev K Gupta, Advanced Biotech Lab, Ipca Laboratories Ltd., Kandivli (W), Mumbai.

E-mail: sanjeev.gupta@ipca.com

Received: October 28, 2019; Published: November 07, 2019

DOI: 10.31080/ASMI.2019.02.0428

Academic institutes and Biopharma Industries are now focusing on novel development to meet current demands for curing several life threatening diseases. Addressing the first generation biologics shortfalls, comes the waves of Next generation bio-therapy, which focuses on various disciplines such as Gene therapy, Stem cell therapy and recombinant antibodies including ADCs. It's potential development can be advantageous for industry as well as patients.

\section{Gene therapy}

The global gene therapy market is estimated to reach $\$ 4,402$ million by 2023 [1]. Gene therapy uses “Genes" to treat or prevent disease by replacing, inactivating, knocking-out a mutated gene or introducing a new gene. The major challenges of gene therapy lies in the gene delivery which includes: Viral Vector system (Adenovirus, lentiviruse etc.) or Non viral vectors system (naked DNA delivery, lipoplexes and polyplexes, oligonucleotides) [2]. Other challenges includes, immune rejection, long term cure, and also ethical issues. High cost of therapy is also one of the major challenges. For instant the gene therapy Glybera, Alipogene tiparvovec (by Uni Quare) first approved in 2012 for hereditary lipoprotein lipase deficiency (LPLD)" came with a \$1.6 million dollar price tag [3].

\section{Cell therapy}

The global cell therapy market size expected to reach $\$ 7.92$ billion by 2025 [4]. Cell therapy marks the amazing progress in the personalized medicine, where cellular material is injected into a patient as treatment to cure the disorders. Cell therapy is clinically used for the treatment of the nervous system, cancers, cardiac disorders, diabetes mellitus, bones and joints disorders, genetic disorders, and wounds of the skin and soft tissues [5].

One of the therapies in limelight for treating cancer is Chimeric Antigen Receptor (CAR) T-cell therapy, in which patient's au- tologous T-cells are genetically modified to express a CAR specific for a tumour antigen, followed by ex-vivo cell expansion and reinfusion into the patients. In addition to technical complexity, the major hurdle in implementation of the therapy is the huge treatment cost: $\$ 475,000$ for tisagenlecleucel and $\$ 373,000$ for axi-cel. Overall incurring the total cost to over $\$ 1.0$ million per patient [6]. This exciting and powerful new therapy requires the formation of new multispecialty medical teams for safe delivery and to successfully manage the resultant complications and research to make it available to people at large population.

Next Generation Antibodies: Next generation antibodies are equally gaining the importance in curing life threatening cancer diseases [7]. These antibodies include:

A. Bi-specific, tri- multi - specific.

B. Smaller antibody fragments or other protein-based scaffolds.

C. Antibody drug conjugates (ADCs): consist of a cytotoxic agent attached to a mAb via chemical linkers. They offer targeted delivery of chemotherapeutic agents directly to the cancerous tissue.

Few examples of commercially available ADCs are brentuximab vedotin (Adcetris ${ }^{\circledR}$; Seattle Genetics) used for the treatment of relapsed or refractory Hodgkin lymphoma and systemic anaplastic large cell lymphoma., ado-trastuzumab emtansine (Kadcyla ${ }^{\circledR}$; Genetech/Roche) for the treatment of HER2-positive.

Among the next generation antibodies, ADCs and Bi- specific antibodies as being the most prevalent, marking the future of antibodies as majorly used for oncology, neurology, ophthalmologic indications. 
Above all the challenges and difficulties, these next generations medicine will mark the future of medicinal world as they are immense powerful and potent to fight any biological target. However, developing these drugs in affordable cost is the biggest challenge at present. Continuous research and technologies are being developed in order to make them commercially available with affordable cost for the betterment of the human health.

Bibliography

1. Gene therapy Market Report- Garima Chandra and Tenzin Kunsel.

2. www.genethrapynet.com

3. Rising tide biology.

4. https://www.grandviewresearch.com/press-release/globalcell-therapy-market

5. Soo Kim DDS and jkaoms "A brief overview of cell therapy and its product". In (2013): 39.5.201.

6. Miliotou AN and Papadopoulou LC. "CAR T-cell Therapy: A New Era in Cancer Immunotherapy". Current Pharmaceutical Biotechnology 19.1 (2018): 5-18.

7. A. Challener. "Witnessing Major Growth in Next-Generation Antibodies Cynthia". BioPharm International 30.4 (2017):1419.

Volume 2 Issue 12 December 2019

C All rights are reserved by Sanjeev K Gupta. 